Article

\title{
Flow of a Dense Suspension Modeled as a Modified Second Grade Fluid
}

\author{
Wei-Tao Wu ${ }^{1}$, Nadine Aubry ${ }^{2}$, James F. Antaki ${ }^{3}$ and Mehrdad Massoudi ${ }^{4, *}$ \\ 1 Department of Aerospace Science and Technology, School of Mechanical Engineering, Nanjing University of \\ Science and Technology, Nanjing 210094, China; weitaowwtw@njust.edu.cn \\ 2 Department of Mechanical and Industrial Engineering, Northeastern University, Boston, MA 02115, USA; \\ n.aubry@northeastern.edu \\ 3 Department of Biomedical Engineering, Cornell University, Ithaca, NY 14853, USA; antaki@cornell.edu \\ 4 U.S. Department of Energy, National Energy Technology Laboratory (NETL), Pittsburgh, PA 15236, USA \\ * Correspondence: mehrdad.massoudi@netl.doe.gov; Tel.: +1-412-386-4975
}

Received: 25 January 2018; Accepted: 31 July 2018; Published: 6 August 2018

\begin{abstract}
In this paper, a simple shear flow of a dense suspension is studied. We propose a new constitutive relationship based on the second grade fluid model for the suspension, capable of exhibiting non-linear effects, where the normal stress coefficients are assumed to depend on the volume fraction of the particles and the shear viscosity depends on the shear rate and the volume fraction. After non-dimensionalizing the equations, we perform a parametric study looking at the effects of the normal stress coefficients and the variable viscosity. The numerical results show that for a certain range of parameters, the particles tend to form a region of high and uniform volume fraction, near the lower half of the flow.
\end{abstract}

Keywords: dense suspension; normal stress effects; second grade fluids; non-Newtonian fluids

\section{Introduction}

Flows of (solid) particles suspended in a fluid present many interesting problems for mathematicians, engineers and physicist. Natural examples of such flows are mudslides, debris flow (Davies, 1986) [1], snow or ice avalanches. Industrial examples of suspensions are slurries, (wet) granular materials, fluidized beds, etc. (see Govier and Aziz (1972) [2], Zandi (1971) [3], Shook and Roco (1991) [4]). In general, these suspensions show non-linear behavior such as normal stress differences, yield stress, discontinuous changes in stresses, etc. [5].to mathematically model these flows, from a continuum mechanics perspective, there are at least two distinct methods; we can look at the suspension as a single continuum where a single constitutive relation for the stress tensor is proposed and the material properties are variables (function of time and space, and perhaps function of shear rate, volume fraction of the solids, etc.), or alternatively, we can look at the two components as two distinct but interacting continua, using the methods of mixture theory, where two equations are needed for the stress tensors and one equation for the interaction forces. For details of the second approach, namely the mixture theory, we refer the readers to papers by Johnson et al. (1991) [6], Massoudi $(2003,2008,2010)[7-9]$. In this paper, we will use the (single component) suspension approach.

From a historical point of view, Reynolds (1885) seems to have been the first scientist who noticed that if a shearing motion in a bed of closely packed particles is to occur, the bed must expand to increase the volume of its voids [10]. He named this phenomenon "dilatancy," which is a manifestation of the normal-stress effects. Reiner $(1945,1948,1958)$ was one of the first who derived a non-Newtonian fluid model to predict dilatancy in wet sand [11-13], even though his model did not show how the volume fraction affects the stresses. Massoudi (2011) [14] modified and generalized this constitutive 
relation, also known as the Reiner-Rivlin [15] fluid model, by suggesting that the shear viscosity would depend on the shear rate and the volume fraction. Later, Wu et al. (2012) considered the shearing motion of such a material [16], and Wu et al. (2013) [17] studied the flow of granular materials down an inclined plane. Their numerical simulations predicted only one non-zero normal stress difference, as is expected in the Reiner-Rivlin fluid type models.

In this short paper, we continue this line of thinking and propose a new constitutive model for the flow of a dense suspension by generalizing the second grade fluid model and assuming that the viscosity depends on the shear rate and the volume fraction, while the normal stress coefficients are only functions of the volume fraction. In the Section 2, the governing equations are introduced. In Section 3, the constitutive equation for the stress tensor is discussed. In Section 4, we show the geometry and the kinematics of the problem and in Section 5, we solve the simple shear flow and perform a parametric study on several different dimensionless numbers.

\section{Governing Equations}

We assume that the motion and the behaviour of the suspension can be described using the traditional methods of continuum mechanics. In the absence of any thermo-chemical and electro-magnetic effects, the basic governing equations are the conservation laws for mass, linear momentum and angular momentum (see Slattery (1999) [18]). As we are only considering a purely mechanical system, the energy equation and the entropy inequality are not considered.

\subsection{Conservation of Mass}

The conservation of mass is:

$$
\frac{\partial \rho}{\partial t}+\operatorname{div}(\rho v)=0
$$

where $\partial / \partial \mathrm{t}$ is the derivative with respect to time, $d i v$ is the divergence operator, $v$ is the velocity vector, and $\rho$ is the density of the suspension given by:

$$
\rho=\rho_{s} \phi
$$

where $\rho_{s}$ is the density of an individual particle (assumed to be constant) and $\phi(x, t)$ is the volume fraction of the particles, where $0 \leq \phi<\phi_{\max }<1 ; \phi$ is an independent kinematical field and is a continuous function of position and time. We will see in Section 4, that the introduction of this kinematical field will present new challenges to us, especially in the specification of the boundary conditions. Furthermore, this term couples the density field and the velocity field and allows for finding particle distribution.

\subsection{Conservation of Linear Momentum}

Let $T$ represent the Cauchy stress tensor, then the balance of linear momentum is:

$$
\rho \frac{d v}{d t}=\operatorname{div} \boldsymbol{T}+\rho \boldsymbol{b}
$$

where $\frac{d v}{d t}=\frac{\partial v}{\partial t}+(g r a d v) v$ and $\boldsymbol{b}$ stands for the body force.

\subsection{Conservation of Angular Momentum}

In absence of couple stresses the Cauchy stress tensor is symmetric, that is,

$$
T=T^{T}
$$

In the next section, we will discuss the constitutive modeling of the stress tensor. 


\section{Constitutive Equation for the Stress Tensor}

In this paper, we model the dense suspension of particles as a single component non-linear fluid-like material. Rajagopal and Massoudi (1990) [19] and Rajagopal et al. (1994) [20] proposed a model, where the Cauchy stress tensor depends on the symmetric part of velocity gradient and another second order tensor related to the density gradient (see also Massoudi and Mehrabadi (2001) [21]). And more recently, Massoudi and Tran (2016) [22] proposed a modified third grade fluid model for a dense suspension of particles.

In this paper, we focus our attention on suspension models where the normal stress effects are present (see Massoudi $(2004,2010)$ [23,24]). One of the simplest constitutive equations capable of describing both of the normal stress effects is the second grade fluid, or the Rivlin-Ericksen fluid of grade two (Rivlin and Ericksen (1955) [25], Truesdell and Noll (1992) [26]). For a second grade fluid, the Cauchy stress tensor is given by:

$$
\mathbf{T}=-p \mathbf{I}+\mu \boldsymbol{A}_{1}+\alpha_{1} A_{2}+\alpha_{2} A_{1}^{2}
$$

where $I$ is the identity tensor, $\mathrm{p}$ is the constraint due to incompressibility (pressure), and the kinematical tensors $A_{1}$ and $A_{2}$ are defined through

$$
\left\{\begin{array}{cccc}
\boldsymbol{A}_{1} & = & \boldsymbol{L}+\boldsymbol{L}^{T} \\
\boldsymbol{A}_{2} & = & \frac{d A_{1}}{d t}+\boldsymbol{A}_{1} \boldsymbol{L}+\boldsymbol{L}^{T} \boldsymbol{A}_{1} \\
\boldsymbol{L} & = & & \text { grad } \boldsymbol{v}
\end{array}\right.
$$

where $L$ is the velocity gradient, $\mu$ is the coefficient of viscosity, $\alpha_{1}$ and $\alpha_{2}$ are material moduli which are commonly referred to as the normal stress coefficients. We modify the traditional second grade fluid model and assume that the suspension can be modeled as

$$
\boldsymbol{T}=\left(p(\phi)+\beta_{r}\right) \boldsymbol{I}+\mu(\phi, \dot{\gamma}) \boldsymbol{A}_{1}+\alpha_{1}(\phi) \boldsymbol{A}_{2}+\alpha_{2}(\phi) \boldsymbol{A}_{1}^{2}
$$

where $\dot{\gamma}=\sqrt{1 / 2 \operatorname{tr}\left(A_{1}^{2}\right)}$ is the shear rate, $\left(p(\phi)+\beta_{r}\right) \boldsymbol{I}$ is the spherical (isotropic part of the) stress tensor (which includes the pressure effects) (see Serrin (1959) [27]);In this paper, we assume $p(\phi)=\beta_{p}$ where $\beta_{r}$ can be a function of the principal invariants of $A_{1}, A_{2}$ and $\phi$, and we also assume that the shear viscosity, $\mu$, depends on the volume fraction and the shear rate and the normal stress coefficients, $\alpha_{1}$ and $\alpha_{2}$, depend on the volume fraction. That is, we assume:

$$
\left\{\begin{array}{l}
\mu=\mu_{r}\left(\phi+\phi^{2}\right) \\
\alpha_{1}=\alpha_{10}\left(\phi+\phi^{2}\right) \\
\alpha_{2}=\alpha_{20}\left(\phi+\phi^{2}\right)
\end{array}\right.
$$

These expressions can be viewed as the Taylor series approximation for the material parameters (Rajagopal, et al. (1994) [20]). Clearly, in Equation (7), the term ' $p$ ' does not have the same meaning or implications as the pressure term in Equation (5). More accurately, as pointed out by Rajagopal (2015) [28], 'p' should be referred to as the "mean value of the stress."

The thermodynamics and stability of fluids of second grade have been studied in detail by Dunn and Fosdick (1974) [29]. They showed that if the fluid is to be thermodynamically consistent, then

$$
\begin{gathered}
\mu \geq 0 \\
\alpha_{1} \geq 0 \\
\alpha_{1}+\alpha_{2}=0
\end{gathered}
$$


It is known that for many non-linear fluids which are assumed to follow Equation (9), the experimental values reported for $\alpha_{1}$ and $\alpha_{2}$ do not satisfy the restriction of Equations (9b) and (9c). In an important paper, Fosdick and Rajagopal (1979) [30] show that irrespective of whether $\alpha_{1}+\alpha_{2}$ is positive, the fluid is unsuitable if $\alpha_{1}$ is negative. For further details on this and other relevant issues in fluids of differential type, we refer the reader to the review article by Dunn and Rajagopal (1995) [31]. In certain applications, where the fluid is known to be shear- thickening (or shear- thinning), then modified (or generalized) forms of the second-grade fluid have been proposed (see Man (1992) [32], Massoudi and Vaidya (2008) [33], Man and Massoudi (2010) [34], Massoudi and Tran (2016) [22]). In this paper, we assume that the viscosity changes with the shear rate and can be modeled as a power-law type relation, where $\mu_{r}$ is given by:

$$
\mu_{r}=\mu_{r 0} \dot{\gamma}^{m}
$$

where $\mu_{r 0}$ is a reference viscosity, assumed to be a constant, $\dot{\gamma}^{m}$ represents the effect of the shear dependency. We should note that when $m<0$, the fluid is shear-thinning, and if $m>0$, the fluid is shear-thickening. The above power-law relation, as is well-known, suffers from the fact that for shear-thinning fluids, the model predicts that the viscosity goes to infinity as the shear rate approaches zero. To overcome this short-coming, we can use an expression of the type $\mu_{r}=\mu_{r 0}\left\lfloor 1+\left(\operatorname{tr} A_{1}^{2}\right)^{1 / 2}\right\rfloor^{m}$ which predicts a constant value for the zero shear rate viscosity. Alternatively, we can use the well-known improvements such as the Carreau-type viscosity models (see Carreau et al. (1997, pp. 39-41) [35]). In this paper, we will use Equation (10).

Based on previous studies (Rajagopal and Massoudi (1990) [19]), we assume

$$
\beta_{p}=\beta_{00}<0
$$

see also $[19,36]$. We also assume that $\beta_{r}$, a parameter which accounts for the compacting (a measure of the rigidity) of the particles, can be given by,

$$
\begin{gathered}
\beta_{r}=h(\phi) C_{r} g(\phi) \\
h(\phi)=\left\{\begin{array}{c}
0, \phi<\phi_{c} \\
1, \phi \geq \phi_{c}
\end{array}\right.
\end{gathered}
$$

where $\phi_{c}$ is the critical value of the volume fraction. In this paper, we assume $g(\phi)=\phi$. In a sense, $C_{r}$ is a material parameter related to how much the particles can be compacted. For very rigid particles, $C_{r}$ is large, ensuring that the local volume fraction of the particles cannot be larger than $\phi_{c}$. We assume that $h(\phi)$ in Equation (12) is a smooth step function, such that

$$
h(\phi)=\frac{1}{1+\exp \left(-2 S\left(\phi-\phi_{c}\right)\right)}
$$

where $S$ is a parameter related to the slope of the step function. For example, if $S$ is chosen as 3000, then $h\left(\phi_{c}-0.001\right) / h\left(\phi_{c}\right)<0.01$ and $h\left(\phi_{c}-0.005\right) \sim o\left(10^{-13}\right)$, therefore $\beta_{r}$ is negligible when $\phi$ is not close to $\phi_{c}$.

Thus the equation for the stress tensor used in our paper, in expanded form is:

$$
\boldsymbol{T}=\left(\beta_{p} \phi+\frac{C_{r}}{1+\exp \left(-2 S\left(\phi-\phi_{c}\right)\right)} \phi\right) \boldsymbol{I}+\mu_{r 0} \dot{\gamma}^{m}\left(\phi+\phi^{2}\right) A_{1}+\alpha_{10}\left(\phi+\phi^{2}\right) A_{2}+\alpha_{20}\left(\phi+\phi^{2}\right) A_{1}^{2}
$$

where $\beta_{p}, C_{r}, \phi_{c}, \mu_{r 0}, m, \alpha_{10}$ and $\alpha_{20}$ are material constants. Note that in the above equation $T$ goes to zero when $\phi \rightarrow 0$, as is expected. Finally, we need to emphasize that Equation (15) being a generalization of the second grade fluid model, contains higher order derivatives of the velocity gradient and as a result, once Equation (15) is substituted in the linear momentum equation, the order 
of the ensuing partial differential equations is one order higher than the Navier-Stokes equations. Therefore, in general, depending on the kinematics of the flow, we may need additional boundary conditions. For a detailed discussion of this issue, we refer the reader to Rajagopal (1995) [37].

\section{Geometry and the Kinematics of the Flow}

The geometry and the kinematics of the flow are shown in Figure 1. Whenever a new model is proposed or developed, it is worthwhile to consider some simple flow situations, usually referred to as viscometric flows (see Truesdell (1974) [38]). This is to see if analytical solutions can be obtained and whether we can gain some physical insights into the nature of the model and perhaps even compare the results with ideal one-dimensional experiments. In this paper, we consider a simple shear flow between two horizontal flat plates (see [16]).

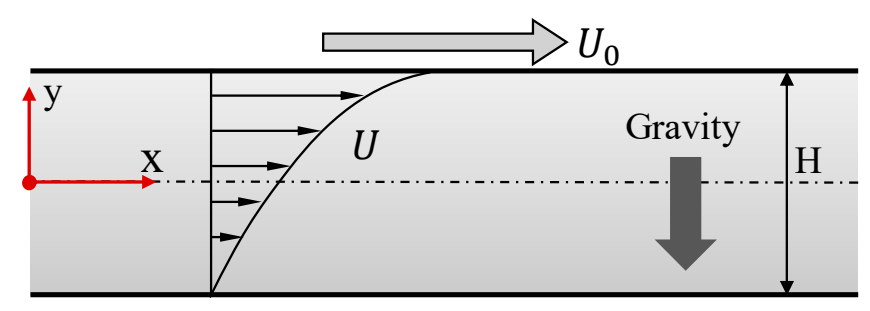

Figure 1. Schematic of the flow.

The non-dimensional form of the conservation of linear momentum is:

$$
\begin{aligned}
\phi\left[\frac{\partial \boldsymbol{V}}{\partial \tau}+(\operatorname{grad} \boldsymbol{V}) \boldsymbol{V}\right] & \\
& =B_{p} \operatorname{grad} \phi+(\operatorname{grad} \phi) h(\phi) B_{r}+\phi \operatorname{grad}\left(h(\phi) B_{r}\right) \\
& +R\left(\Gamma^{m} \operatorname{div}\left(\left(\phi+\phi^{2}\right) \boldsymbol{A}_{1}\right)+\boldsymbol{A}_{1}\left(\phi+\phi^{2}\right) \operatorname{grad} \Gamma^{m}\right)+\frac{1}{F r} \phi \\
& +M_{1} \operatorname{div}\left(\left(\phi+\phi^{2}\right) \boldsymbol{A}_{2}\right)+M_{2} \operatorname{div}\left(\left(\phi+\phi^{2}\right) \boldsymbol{A}_{1}^{2}\right)
\end{aligned}
$$

where we have used the following reference parameters:

$$
\begin{gathered}
Y=\frac{y}{H} ; X=\frac{x}{H} ; V=\frac{v}{u_{0}} ; \tau=\frac{t u_{0}}{H} ; \\
\text { where } \\
\operatorname{div}^{*}(\cdot)=H \operatorname{div}(\cdot) ; \operatorname{grad}^{*}(\cdot)=H \operatorname{Hrad}(\cdot) ; L^{*}=\operatorname{grad}^{*} \boldsymbol{V} \\
A_{1}{ }^{*}=L^{*}+L^{* T} ; A_{2}{ }^{*}=\frac{d A_{1}{ }^{*}}{d \tau}+A_{1}{ }^{*} L^{*}+L^{* T} A_{1}{ }^{*} \\
B_{p}=\frac{\beta_{p}}{\rho_{s} u_{0}^{2}} ; B_{r}=\frac{C_{r}}{\rho_{s} u_{0}^{2}} ; F r=\frac{u_{0}^{2}}{H g} ; R=\frac{\mu_{r 0}}{\rho_{s} u_{0}{ }^{1-m} H^{1+m}} ; \\
\Gamma=\frac{H \gamma}{u_{0}} ; M_{1}=\frac{\alpha_{10}}{\rho_{s} H^{2}} ; M_{2}=\frac{\alpha_{20}}{\rho_{s} H^{2}} ;
\end{gathered}
$$

where $H$ is a reference length, for example, the distance between the two plates, and $u_{0}$ is a reference velocity, for example the (shearing) velocity of the upper plate, $U_{0}$. In Equation (16) the asterisks have been dropped for simplicity. Among the above dimensionless numbers, $M_{1}$ and $M_{2}$ are related to the normal stress coefficients, $B_{p}$ is related to the compressibility factor (the pressure term), $B_{r}$ is related to the compacting effect of the particles. $B_{p}$ and $B_{r}$ are always less than zero, indicating that with compression there is a tendency for densification. $F r$ is the Froude number, $R$ is related to the viscous effects (similar to the Reynolds number), and $\Gamma$ is the dimensionless shear rate parameter.

For a simple shear flow, we assume:

$$
\boldsymbol{v}=U(Y) \boldsymbol{e}_{x} ; \phi=\phi(y)=\rho(y) / \rho_{0}
$$


where $\boldsymbol{e}_{x}$ is the unit vector along the $x$ direction, then using Equation (18), we have,

$$
\begin{gathered}
\boldsymbol{D}=2 A_{1}=\frac{1}{2}\left(g r a d v+(g r a d v)^{T}\right)=\frac{1}{2}\left[\begin{array}{ccc}
0 & U^{\prime} & 0 \\
U^{\prime} & 0 & 0 \\
0 & 0 & 0
\end{array}\right] \\
\operatorname{tr} \boldsymbol{D}=\operatorname{div} v=0 \\
A_{1}=\left[\begin{array}{ccc}
0 & U^{\prime} & 0 \\
U^{\prime} & 0 & 0 \\
0 & 0 & 0
\end{array}\right] ; A_{2}=2\left[\begin{array}{ccc}
0 & 0 & 0 \\
0 & U^{\prime 2} & 0 \\
0 & 0 & 0
\end{array}\right]
\end{gathered}
$$

where $\boldsymbol{D}$ is the symmetric part of the velocity gradient. In the above equations, prime designates the derivative with respect to $y$. Substituting (18)-(21) into (16), the governing equations are simplified and we obtain a system of non-linear ordinary differential equations.

The momentum equation in the $x$-direction is:

$$
R\left|U^{\prime}\right|^{m}(1+2 \phi) \phi^{\prime} U^{\prime}+R\left(\phi+\phi^{2}\right)(1+m)\left|U^{\prime}\right|^{m} U^{\prime \prime}=0
$$

And in the $y$-direction is:

$$
B_{p} \phi^{\prime}+B_{r} \phi^{\prime} h(\phi)+B_{r} \phi h^{\prime}(\phi)+\left(2 M_{1}+M_{2}\right) \phi^{\prime} U^{\prime 2}(1+2 \phi)+2\left(2 M_{1}+M_{2}\right)\left(\phi+\phi^{2}\right) U^{\prime} U^{\prime \prime}-\frac{1}{F r} \phi=0
$$

From Equation (22) we can see that dimensionless number, $R$, can be canceled for this problem. The normal stress differences for this problem are,

$$
\begin{gathered}
T_{x x}-T_{y y}=-2 M_{1}\left(\phi+\phi^{2}\right) U^{\prime 2} \\
T_{y y}-T_{z z}=2 M_{1}\left(\phi+\phi^{2}\right) U^{\prime 2}+M_{2}\left(\phi+\phi^{2}\right) U^{\prime 2}
\end{gathered}
$$

From Equations (22) and (23), it is clear that we need two boundary conditions for $U$ and one boundary condition for $\phi$. We use the no-slip condition at both boundaries for the velocity:

$$
U(Y=0)=0 ; U(Y=1)=1
$$

It is possible that the particles may slip at the walls (see Kim and Rosato (1994) [39], Zhao and Massoudi (2014) [40]). For volume fraction, $\phi$, the appropriate boundary condition may be given as an average value defined in an integral form:

$$
\int_{-1}^{1} \phi d Y=N
$$

or $\phi$ could be prescribed at $Y=-1$ :

$$
\phi \rightarrow \Theta \text { as } Y \rightarrow-1
$$

where $\Theta$ is the value of the volume fraction at the boundary. In this paper, the condition of the average volume fraction is used (Equation (27)). Note that since we are including the effect of gravity, we do not use the symmetry boundary condition for the volume fraction, to allow for the settling of the particles.

\section{Results and Discussion}

The system of the non-linear ordinary differential Equations (22) and (23) with the boundary conditions (26) and (27) are solved using the MATLAB solver bvp4c, which is a collocation boundary value problem solver [41]. The step size is automatically adjusted by the solver. The default relative tolerance for the maximum residue is 0.001 . The boundary conditions for the average volume fraction 
are numerically satisfied by using the shooting method. Since the model we have proposed is a new model, there are no experimental data available to use for the material properties. Therefore, we will perform a parametric study for a limited range of the dimensionless numbers specified in Table 1.

Table 1. The studied dimensionless numbers.

\begin{tabular}{cc}
\hline Dimensionless Numbers & Studied Value \\
\hline$m$ & $-0.5,0.0,1.0$ \\
$\left(2 M_{1}+M_{2}\right)$ & $0,1,10$ \\
$B_{p}$ & $-0.5,-2.0,-10.0$ \\
$B_{r}$ & $0.0,-0.1,-10$ \\
$\phi_{c}$ & $0.5,0.6,0.68$ \\
$F r$ & $0.25,0.5,2.5$ \\
$N$ & $0.2,0.35,0.4,0.5$ \\
$R$ & 1.0 \\
\hline
\end{tabular}

\subsection{The Effect of the Compacting of the Particles}

First, we consider the isotropic (spherical) part of the stress tensor due to the particle contact. Recall that $B_{r}$ is related to the level of the rigidity of the particles and $\phi_{c}$ is related to the maximum packing of the particles. Figure 2 shows the effect of $B_{r}$ on the volume fraction profiles. The influence of this dimensionless number on the velocity profiles, for the range of dimensionless parameters selected in this paper, is negligible and as a result we do not show it. The value of $B_{r}$ is always equal to or less than zero which ensures that with compression, the density increases. We can see that due to the effect of the gravity, the particles tend to accumulate near the bottom plate. Figure 2 shows that as $B_{r}$ changes, near the bottom plate the particles cannot be further compacted once the volume fraction has reached $\phi_{c}$; as a result, a high concentration layer/region seems to be formed. In this region, the volume fraction is uniform; while from the edge of this region, along the $y$-direction (see Figure 1 for the definition of the coordinates) the gradient of the volume fraction profile suddenly becomes large. When $B_{r}$ is small, near the lower plate the local volume fraction continues to increase; from Figure 2 we can see that the spherical stress, $\beta_{r}$, is important even when the magnitude of $B_{r}$ is small, see the cases for $B_{r}=0$ and $B_{r}=-0.1$.

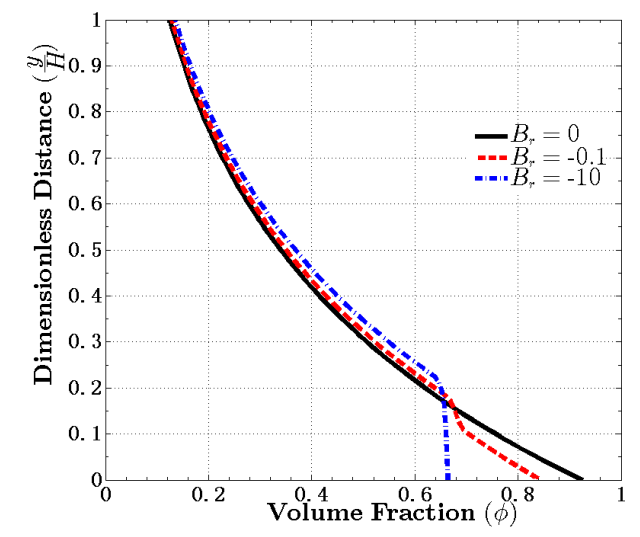

Figure 2. The effect of $B_{r}$ on the volume fraction profile, when $R=1, m=1,\left(2 M_{1}+M_{2}\right)=1$, $B_{p}=-1, \phi_{c}=0.68, S=100, F r=0.5, N=0.4$.

From Figure 3, we see that as $\phi_{c}$ decreases, near the bottom plate the thickness of the high concentration layer/region increases; in this region, the local volume fraction is uniform and close to $\phi_{c}$. As Figure 3a shows, when $\phi_{c}$ decreases, near the bottom plate the velocity increases and the profile becomes more linear in the region with high volume fraction. This may be due to the fact that when $\phi_{c}$ 
is small, the volume fraction distribution is more uniform, which leads to a more uniform distribution of the viscosity.

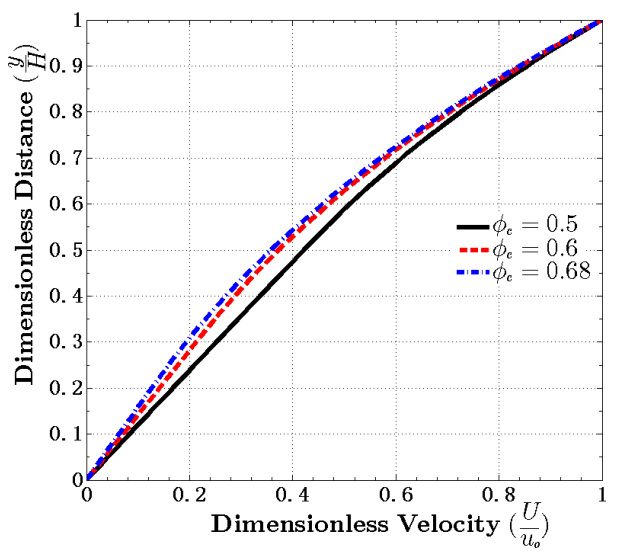

(a)

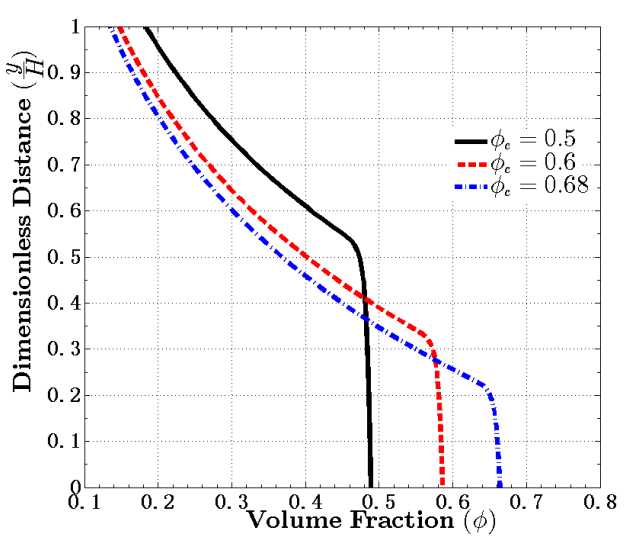

(b)

Figure 3. The effect of $\phi_{c}$ on the velocity profile (a) and the volume fraction profile (b), when $R=1$, $m=1,\left(2 M_{1}+M_{2}\right)=1, B_{p}=-1, B_{r}=-10, S=100, F r=0.5, N=0.4$.

\subsection{Effects of the Normal Stress Coefficients and the (Variable) Viscosity}

In our model, the normal stress differences are related to the dimensionless parameters $M_{1}$ and $M_{2}$. Their effect appears as the combined parameter $\left(2 M_{1}+M_{2}\right)$, see Equations (24) and (25). From Figure $4 \mathrm{~b}$, we can see that as $\left(2 M_{1}+M_{2}\right)$ increases, near the upper plate, the volume fraction increases; this indicates that more particles have moved to that region. While near the bottom plate, the high concentration region tends to disappear. The velocity increases as $\left(2 M_{1}+M_{2}\right)$ increases. Recall that $B_{p}$ is a dimensionless parameter related to the pressure. As Figure $5 \mathrm{~b}$ shows, when the magnitude of $B_{p}$ increases, the concentration profiles become more uniform and linear-like, which indicates that the effects of gravity $(F r)$, spherical stress $\left(B_{r}\right)$, and the normal stress differences $\left(2 M_{1}+M_{2}\right)$ are less important (see Equation (23)). As $B_{p}$ increases, the volume fraction distribution becomes more uniform and the velocity profiles become more linear.

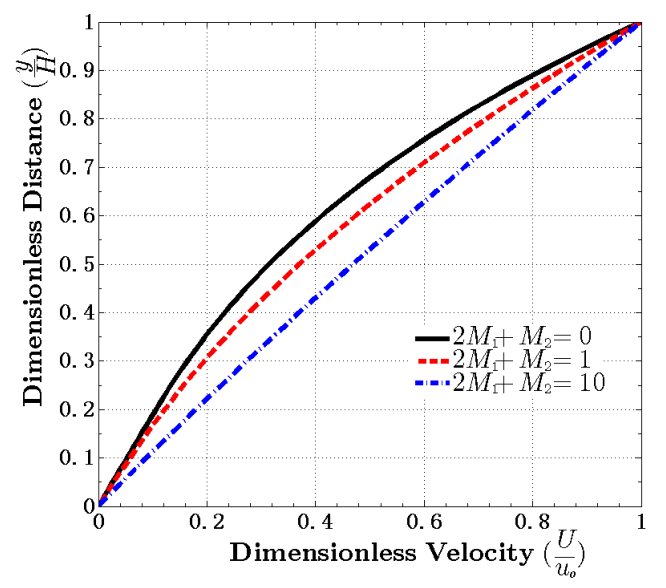

(a)

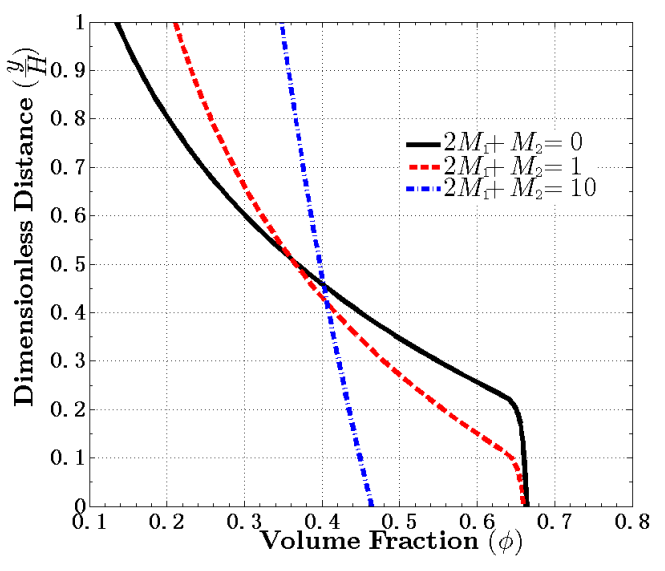

(b)

Figure 4. The effect of $\left(2 M_{1}+M_{2}\right)$ on the velocity profile (a) and the volume fraction profile (b), when $R=1, m=1, B_{p}=-1, B_{r}=-10, \phi_{c}=0.68, S=100, F r=0.5, N=0.4$. 


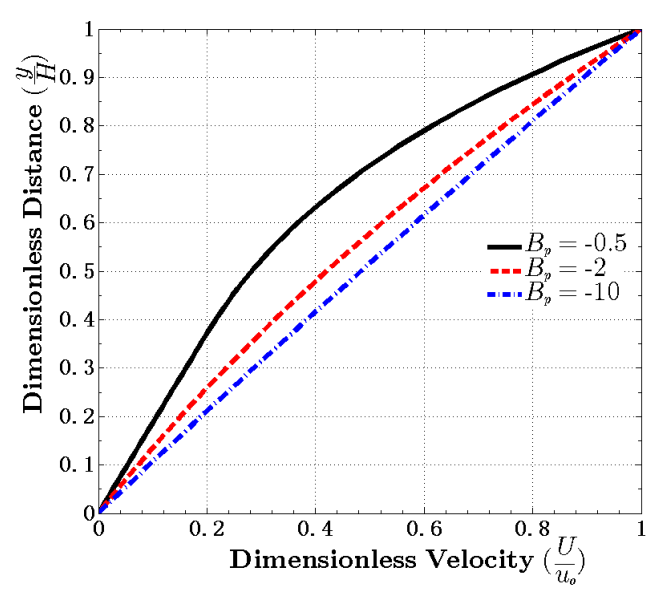

(a)

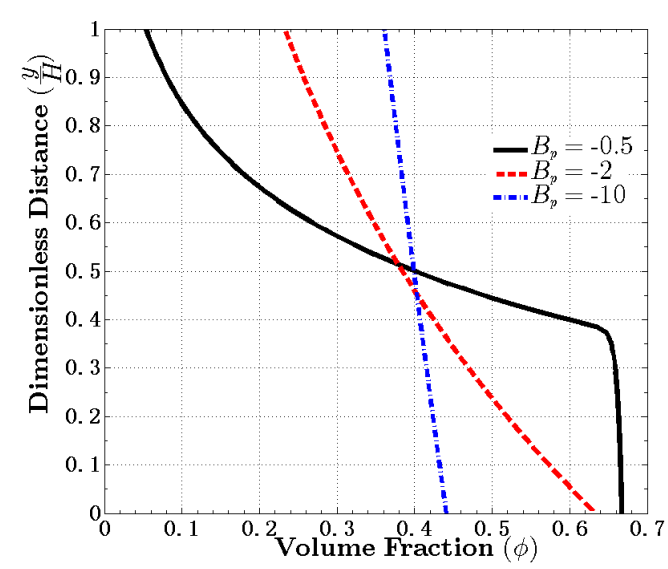

(b)

Figure 5. The effect of $B_{p}$ on the velocity profile (a) and the volume fraction profile (b), when $R=1$, $m=0.5,\left(2 M_{1}+M_{2}\right)=1, B_{r}=-10, \phi_{c}=0.68, S=100, F r=0.5, N=0.4$.

Another important quantity of interest is the (dimensionless) wall shear stress, $\left|\tau_{w}\right|$ which is related to the skin-friction coefficient [42]. In this problem, $\left|\tau_{w}\right|$ has the following expression:

$$
\left|\tau_{w}\right|=\left.R\left(\phi+\phi^{2}\right)\left|U^{\prime}\right|^{m} \sqrt{2 U^{\prime 2}}\right|_{w}
$$

Table 2 shows the effect of the normal stresses, $\left(2 M_{1}+M_{2}\right)$ and $B_{p}$, on the wall shear stress. As the effect of the normal stresses increases, the wall shear stress also increases.

Table 2. The effect of the normal stresses $\left(2 M_{1}+M_{2}\right)$ and $B_{p}$ on the wall shear stress.

\begin{tabular}{cccc}
\hline$\left(\mathbf{2 M}_{\mathbf{1}}+\boldsymbol{M}_{\mathbf{2}}\right)$ & $\left|\boldsymbol{\sigma}_{w}\right|$ & $\boldsymbol{B}_{\boldsymbol{p}}$ & $\left|\boldsymbol{\sigma}_{\boldsymbol{w}}\right|$ \\
\hline 0 & 0.6010 & -0.25 & 0.4527 \\
1 & 0.6886 & -2.00 & 0.7343 \\
10 & 0.7874 & -10.0 & 0.7894 \\
\hline
\end{tabular}

In our model, $m$ is the parameter related to the effects of variable (shear-dependent) viscosity. Recall that $m>0$ and $m<0$ imply shear-thickening and shear-thinning behavior, respectively. Figure 6 shows the effect of $m$ on the velocity and the volume fraction distributions. As the magnitude of $m$ increases, the volume fraction distribution becomes more non-uniform and the high concentration region near the lower plate becomes more significant when $m$ is positive. As $m$ increases, the velocity decreases.

\subsection{Effects of Gravity and the Bulk Volume Fraction}

Figure 7 shows the effect of $\mathrm{Fr}$ number, which is the ratio of the inertial forces to the gravitational force. As Fr number decreases, that is, lower flow rates, more particles seem to accumulate in the lower half of the flow, and therefore the volume fraction near the bottom plate increases. When Fr number is large, the velocity seems to be more linear. The effect of the bulk volume fraction on the flow is shown in Figure 8. The thickness of the high concentration region increases as $N$ increases. Increasing $N$ seems to lead to a more linear-like velocity distribution and more uniform volume fractions. 


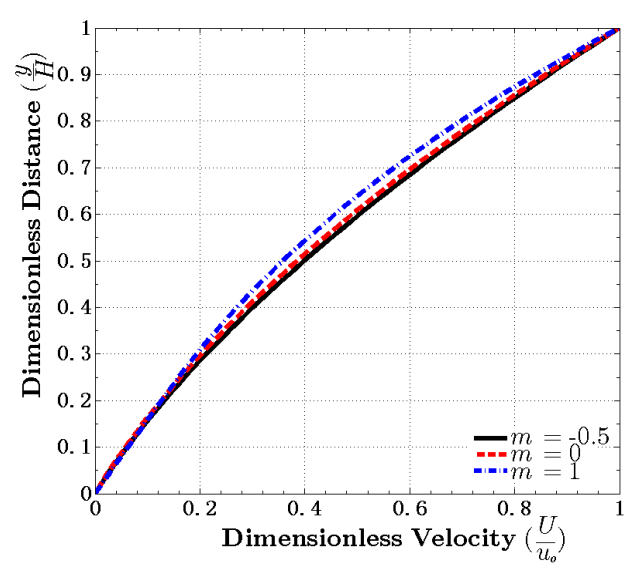

(a)

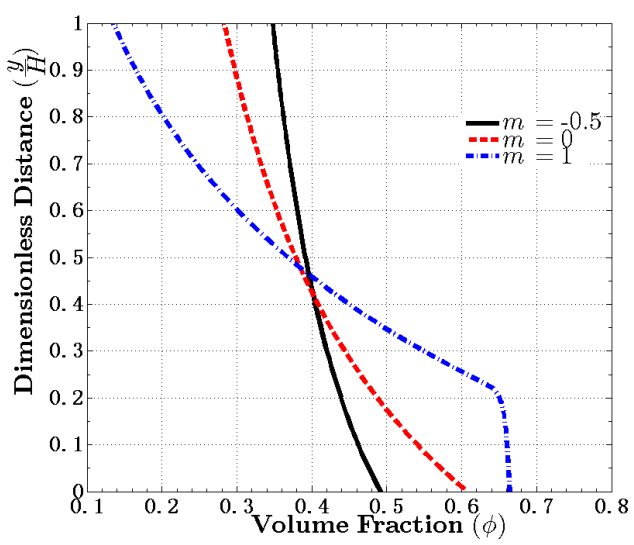

(b)

Figure 6. The effect of $m$ on the velocity profile (a) and the volume fraction profile (b), when $R=1$, $\left(2 M_{1}+M_{2}\right)=1, B_{p}=-1, B_{r}=-10, \phi_{c}=0.68, S=100, F r=0.5, N=0.4$.

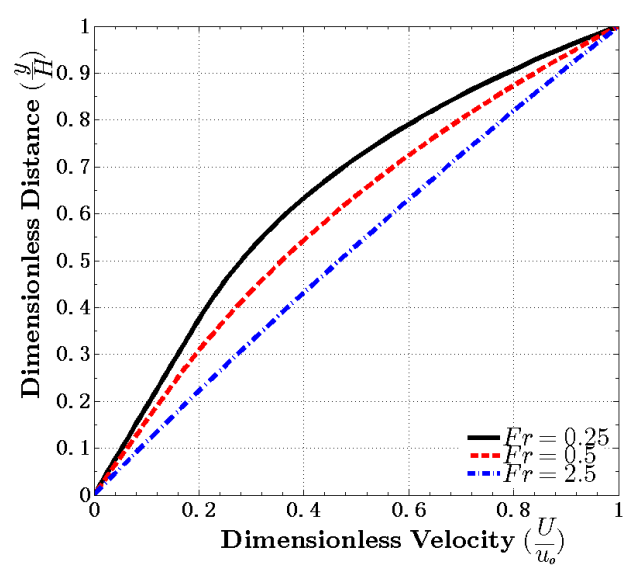

(a)

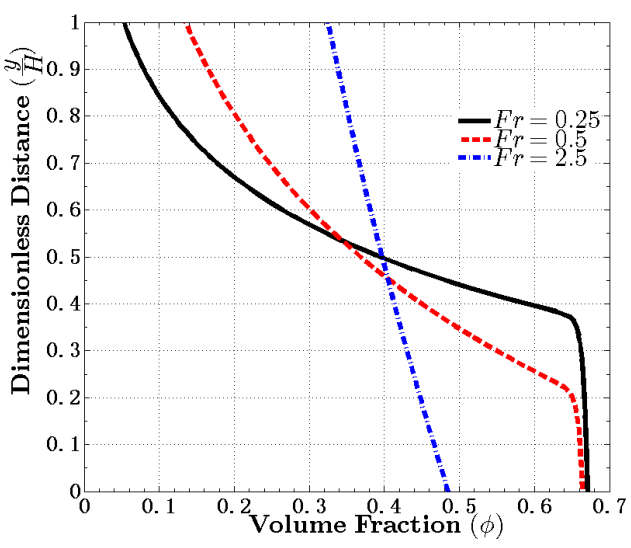

(b)

Figure 7. The effect of $\mathrm{Fr}$ number on the velocity profile (a) and the volume fraction profile (b), when $R=1, m=1,\left(2 M_{1}+M_{2}\right)=1, B_{p}=-1, B_{r}=-10, \phi_{c}=0.68, S=100, N=0.4$.

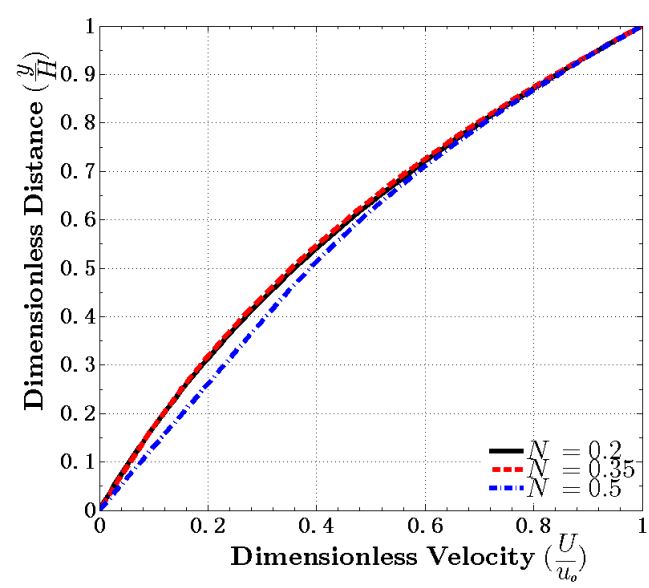

(a)

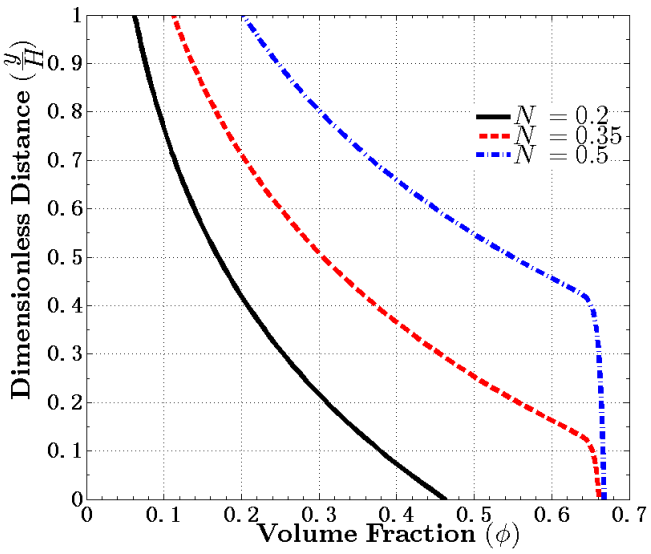

(b)

Figure 8. The effect of $N$ on the velocity profile (a) and the volume fraction profile (b), when $R=1$, $m=1,\left(2 M_{1}+M_{2}\right)=1, B_{p}=-1, B_{r}=-10, \phi_{c}=0.68, S=100, F r=0.5$. 


\section{Conclusions}

In this paper, we study the simple shear flow of a non-linear fluid (a dense suspension of particles in a liquid) between two horizontal plates. The fluid is modeled as a modified second grade fluid. Important rheological properties such as the shear rate and the volume fraction dependency of the viscosity and the normal stress coefficients are considered. Through numerical simulations, we find that for a certain range of parameters, the particles tend to accumulate near the bottom plate where a region with high concentration of particles is formed. In addition, the results also show that as the normal stress effects increase, there is an increase in the value of the wall shear stress. As the model developed in this paper is a new model, there are no experimental data with which we can compare the results of our studies.

Author Contributions: Most of the mathematical formulation was primarily done by M.M., part of the derivation and the numerical work were done by W.-T.W. All authors contributed to the writing of the paper.

Funding: This research received no external funding.

Conflicts of Interest: The authors declare no conflict of interest.

\section{References}

1. Davies, T.R.H. Large debris flows: A macro-viscous phenomenon. Acta Mech. 1986, 63, 161-178. [CrossRef]

2. Govier, G.W.; Aziz, K. The Flow of Complex Mixtures in Pipes; Van Nostrand Reinhold Company: New York, NY, USA, 1972; Volume 469.

3. Zandi, I. Advances in solid-liquid flow in pipes and its application. In International Symposium in Solid-Liquid Flow in Pipe and Removal of Solid Waste (1968: University of Pennsylvania); Pergamon Press: Oxford, UK, 1971.

4. Shook, C.A.; Roco, M.C. Slurry flow: Principles and Practice; Butterworth-Heinemann: Boston, MA, USA, 1991.

5. Savage, S.B.; Jeffrey, D.J. The stress tensor in a granular flow at high shear rates. J. Fluid Mech. 1981, 110, 255-272. [CrossRef]

6. Johnson, G.; Massoudi, M.; Rajagopal, K.R. Flow of a fluid-Solid mixture between flat plates. Chem. Eng. Sci. 1991, 46, 1713-1723. [CrossRef]

7. Massoudi, M. Constitutive relations for the interaction force in multicomponent particulate flows. Int. J. Non. Linear. Mech. 2003, 38, 313-336. [CrossRef]

8. Massoudi, M. A note on the meaning of mixture viscosity using the classical continuum theories of mixtures. Int. J. Eng. Sci. 2008, 46, 677-689. [CrossRef]

9. Massoudi, M. A Mixture Theory formulation for hydraulic or pneumatic transport of solid particles. Int. J. Eng. Sci. 2010, 48, 1440-1461. [CrossRef]

10. Reynolds, O. On the dilatancy of media composed of rigid particles in contact. With experimental illustrations. Philos. Mag. Ser. 1885, 20, 469-481. [CrossRef]

11. Reiner, M. A mathematical theory of dilatancy. Am. J. Math. 1945, 67, 350-362. [CrossRef]

12. Reiner, M. Elasticity beyond the elastic limit. Am. J. Math. 1948, 70, 433-446. [CrossRef]

13. Reiner, M. Rheology. In Handbuch Der Physik; Flugge, S., Ed.; Springer: Berlin, Germany, 1958; Volume VI.

14. Massoudi, M. A generalization of Reiner's mathematical model for wet sand. Mech. Res. Commun. 2011, 38, 378-381. [CrossRef]

15. Rivlin, R.S. The hydrodynamics of non-Newtonian fluids. I. In Proceedings of the Royal Society of London A: Mathematical, Physical and Engineering Sciences; The Royal Society: London, UK, 1948; Volume 193, pp. 260-281.

16. Wu, W.-T.; Aubry, N.; Massoudi, M. Flow of Granular Materials Modeled as a Generalized Reiner-Rivlin Type Fluid. In ASME 2012 International Mechanical Engineering Congress and Exposition; American Society of Mechanical Engineers: New York, NY, USA, 2012; pp. 1077-1083.

17. Wu, W.T.; Aubry, N.; Massoudi, M. Flow of granular materials modeled as a non-linear fluid. Mech. Res. Commun. 2013, 52, 62-68. [CrossRef]

18. Slattery, J.C. Advanced Transport Phenomena; Cambridge University Press: Cambridge, UK, 1999. 
19. Rajagopal, K.R.; Massoudi, M. A Method for Measuring the Material Moduli of Granular Materials: Flow in an Orthogonal Rheometer (No. DOE/PETC/TR-90/3); USDOE Pittsburgh Energy Technology Center: Pittsburgh, PA, USA, 1990.

20. Rajagopal, K.R.; Massoudi, M.; Wineman, A.S. Flow of granular materials between rotating disks. Mech. Res. Commun. 1994, 21, 629-634. [CrossRef]

21. Massoudi, M.; Mehrabadi, M.M. A continuum model for granular materials: Considering dilatancy and the Mohr-Coulomb criterion. Acta Mech. 2001, 152, 121-138. [CrossRef]

22. Massoudi, M.; Tran, P.X. The Couette-Poiseuille flow of a suspension modeled as a modified third-grade fluid. Arch. Appl. Mech. 2016, 86, 921-932. [CrossRef]

23. Massoudi, M. Constitutive modelling of flowing granular materials: A continuum approach. In Granular Materials: Fundamentals and Applications; Antony, S.J., Hoyle, W., Ding, Y., Eds.; RSC: Cambridge, UK, 2004; Volume 63.

24. Massoudi, M. Mathematical Modelling of Granular materials. In Rheology of Complex Fluids; Deshpande, A.P., Krishnan, J.M., Kumar, P.B.S., Eds.; Springer: New York, NY, USA, 2010; pp. 219-245.

25. Rivlin, R.S.; Ericksen, J.L. Stress-deformation relations for isotropic materials. J. Ration. Mech. Anal. 1955, 4, 323-425. [CrossRef]

26. Truesdell, C.; Noll, W. The Nonlinear Field Theories of Mechanics; Springer: Berlin, Germany, 1992.

27. Serrin, J. The derivation of stress-deformation relations for a Stokesian fluid. J. Math. Mech. 1959, 459-469. [CrossRef]

28. Rajagopal, K.R. Remarks on the notion of "pressure". Int. J. Non. Linear. Mech. 2015, 71, 165-172. [CrossRef]

29. Dunn, J.E.; Fosdick, R.L. Thermodynamics, stability, and boundedness of fluids of complexity 2 and fluids of second grade. Arch. Ration. Mech. Anal. 1974, 56, 191-252. [CrossRef]

30. Fosdick, R.L.; Rajagopal, K.R. Anomalous features in the model of "second order fluids". Arch. Ration. Mech. Anal. 1979, 70, 145-152. [CrossRef]

31. Dunn, J.E.; Rajagopal, K.R. Fluids of differential type: Critical review and thermodynamic analysis. Int. J. Eng. Sci. 1995, 33, 689-729. [CrossRef]

32. Man, C.-S. Nonsteady channel flow of ice as a modified second-order fluid with power-law viscosity. Arch. Ration. Mech. Anal. 1992, 119, 35-57. [CrossRef]

33. Massoudi, M.; Vaidya, A. On some generalizations of the second grade fluid model. Nonlinear Anal. Real World Appl. 2008, 9, 1169-1183. [CrossRef]

34. Man, C.-S.; Massoudi, M. On the thermodynamics of some generalized second-grade fluids. Contin. Mech. Thermodyn. 2010, 22, 27-46. [CrossRef]

35. Carreau, P.J.; De Kee, D.; Chhabra, R.J. Rheology of Polymeric Systems; Hanser/Gardner Publications: Cincinnati, OH, USA, 1997.

36. Rajagopal, K.R.; Troy, W.; Massoudi, M. Existence of solutions to the equations governing the flow of granular materials. Eur. J. Mech. B. Fluids 1992, 11, 265-276.

37. Rajagopal, K.R. On boundary conditions for fluids of the differential type. In Navier-Stokes Equations and Related Nonlinear Problems; Springer: New York, NY, USA, 1995; pp. 273-278.

38. Truesdell, C. The meaning of viscometry in fluid dynamics. Annu. Rev. Fluid Mech. 1974, 6, 111-146. [CrossRef]

39. Rosato, A.D.; Kim, H. Particle dynamics calculations of wall stresses and slip velocities for Couette flow of smooth inelastic spheres. Contin. Mech. Thermodyn. 1994, 6, 1-20. [CrossRef]

40. Zaho, X.; Massoudi, M. Flow of Granular Materials with Slip Boundary Condition: A Continuum-Kinetic Theory Approach. Appl. Math. Comput. 2014, 242, 518-527.

41. Mu-Analysis and Synthesis Toolbox User's Guide; The Mathworks, Inc.: Natick, MA, USA, 1998; Volume 5.

42. Gupta, G.; Massoudi, M. Flow of a generalized second grade fluid between heated plates. Acta Mech. 1993, 99, 21-33. [CrossRef]

(C) 2018 by the authors. Licensee MDPI, Basel, Switzerland. This article is an open access article distributed under the terms and conditions of the Creative Commons Attribution (CC BY) license (http:/ / creativecommons.org/licenses/by/4.0/). 